\title{
Effect of siRNA-induced inhibition of IL-6 expression in rat cerebral gliocytes on cerebral edema following traumatic brain injury
}

\author{
BIN XU ${ }^{1}$, DONG-MING YU ${ }^{1}$ and FU-SHENG LIU ${ }^{2}$ \\ ${ }^{1}$ Department of Emergency, Beijing Tiantan Hospital, Capital Medical University; ${ }^{2}$ Brain Tumor Research Center, \\ Beijing Neurosurgical Institute, Beijing Tiantan Hospital, Capital Medical University, Beijing 100050, P.R. China
}

Received October 13, 2013; Accepted April 25, 2014

DOI: $10.3892 / \mathrm{mmr} .2014 .2462$

\begin{abstract}
The present study aimed to investigate the effect of RNA interference (RNAi) on the inhibition of interleukin (IL)-6 expression in rat cerebral gliocytes in vitro and rat cerebral traumatic tissues in vivo, as well as the effect of RNAi on cerebral edema. pSUPER vectors containing IL-6 small hairpin RNA (pSUPER-IL-6 1-5) were designed, constructed and transfected into C6 rat glioma cells using cationic liposomes. ELISA was used to select the plasmid with the strongest interference effect. A freefall method was used to generate a rat brain injury model and rats were randomly divided into treatment, empty plasmid and control groups ( $n=14 /$ group). IL-6 levels, water content and sodium content were determined in the brain tissues at 24 and $72 \mathrm{~h}$ post-injury. pSUPER-IL- 6 was effectively transfected into C6 cells and was found to inhibit the expression of IL-6 rather than IL-8. The pSUPER-IL-6 1 vector was most effective in inducing RNAi. In vivo, IL-6 levels were observed to be lowest in the interference group and there were statistically significant differences in water and sodium content among the experimental groups $(\mathrm{P}<0.05)$. RNAi was found to inhibit IL-6 expression in vivo and in vitro in rat cerebral gliocytes, and the reduction of the IL-6 levels was found to reduce post-traumatic cerebral edema.
\end{abstract}

Correspondence to: Dr Dong-Ming Yu, Department of Emergency, Beijing Tiantan Hospital, Capital Medical University, 6 Tiantan Xili, Dongcheng, Beijing 100050, P.R. China

E-mail: dongmingyucn@126.com

Dr Fu-Sheng Liu, Brain Tumor Research Center, Beijing Neurosurgical Institute, Beijing Tiantan Hospital, Capital Medical University, 6 Tiantan Xili, Beijing 100050, P.R. China

E-mail: fsliucn@126.com

Key words: interleukin-6, RNA interference, brain trauma, cerebral edema

\section{Introduction}

Traumatic brain injury (TBI) is one of the leading causes of injury-associated death and disability, particularly in young individuals (1). Brain damage following traumatic injury may be a result of direct mechanisms, for example immediate mechanical disruption of the brain tissue and primary injury, or indirect mechanisms, such as secondary or delayed injury. Secondary mechanisms involve the initiation of an acute inflammatory response, including breakdown of the blood-brain barrier (BBB), edema and swelling, infiltration of peripheral blood cells and activation of resident immunocompetent cells, as well as the intrathecal release of numerous immune mediators, such as interleukins (ILs) and chemotactic factors (2). Pro-inflammatory genes, including IL-1 $\beta$, tumor necrosis factor (TNF)- $\alpha$ and IL-6, have been reported to be differentially regulated following TBI (3-6). Furthermore, high levels of IL-6 and soluble IL-6 receptor (sIL-6R) have been reported in the cerebrospinal fluid (CSF) of patients with cerebral trauma (7).

In response to injury, IL- 6 acts as an important mediator for the initiation and progression of post-traumatic inflammation, causing additional cell death and neurological dysfunction. IL-6 may also facilitate reparative processes (8). Overproduction of IL-6 in the central nervous system (CNS) has been reported to increase the production of inflammatory cytokines, and thus, IL-6 may have a proinflammatory and detrimental role when dysregulated in the CNS. IL-6 is an essential cytokine required for normal brain function, not only during injury, but also under normal conditions (9). IL-6 has been shown to exert numerous effects, both beneficial and destructive, on CNS cells. However, downregulation of IL-6 is required to maintain its beneficial functions and prevent its potentially detrimental effects (10).

Anti-IL-6 strategies represent an attractive therapeutic option in several diseases, including B-cell neoplasia, osteoporosis and autoimmunity $(11,12)$. Thus, IL- 6 may be a target for therapeutic intervention. Although the neutralization of IL-6 activity using monoclonal antibodies (mAbs) or receptor antagonists is beneficial for the treatment of immune and neoplastic diseases, the necessity for continuous delivery of these anticytokine agents poses considerable practical limitations. Furthermore, systemic administration of antibodies may compromise the host's 
capacity to fight infection. In addition, therapeutic attempts in humans have shown that the administration of injectable doses of anti-IL-6 mAbs does not provide efficient neutralization of IL-6 in vivo and the use of humanized antibodies in the clinic is hindered by a combination of factors, including their short half-life, rapid blood clearance, immunogenicity and high cost. Therefore, alternative approaches are required.

RNA interference (RNAi) is a sequence-specific, post-transcriptional gene silencing mechanism (13). RNAi-induced gene silencing involves processing long double-stranded (ds) RNA into 19-21 nucleotide RNA known as short interfering (si) RNA, which promotes the enzymatic cleavage of complementary mRNA. RNAi is a powerful and widely used tool for the analysis of gene function in invertebrates and vertebrates (14). However, the effectiveness of RNAi-induced protein expression attenuation depends on the efficiency of the cellular uptake of the dsRNA, the half-life of the dsRNA inside the cells and the half-life of the protein to be silenced. The gene silencing induced by siRNA is often poor, which limits its application. The pSUPER vector system has been reported to be capable of directing the synthesis of siRNA in mammalian cells and to induce efficient, sustained and specific knockdown of target gene expression (15). Of note, this vector-based siRNA system has been shown to stably repress gene expression.

In the present study, vector-based siRNA was used to knockdown IL-6 gene expression in C6 rat glioma cells. Furthermore, this vector was used to downregulate IL-6 expression in rat brains exhibiting stab wound injury. The effect of pSUPER-IL-6-induced siRNA expression on the downregulation of endogenous, lipopolysaccharide (LPS)-induced IL-6 expression was investigated in C6 cells. In addition, the effect of small hairpin (sh)RNA administration on the reduction of IL-6 expression, inhibition of inflammation and elimination of brain edema was investigated in TBI rats in vivo.

\section{Materials and methods}

Animals. A total of 42 12-week-old, male Sprague-Dawley rats weighing 250-300 g were purchased from the Center for Experimental Animals, Chinese Academy of Medical Sciences (Beijing, China). The animals were maintained at the animal facility of the Beijing Neurosurgical Institute (Beijing, China) according to the institute's guidelines. The study was approved by Laboratory Animal Welfare Ethics Committee of Beijing Neurosurgical Institute (Beijing, China).

Cell culture. C6 rat glioma cells of Rattus norvegicus origin, as well as C6-green fluorescent protein (GFP) cells (Beijing Neurosurgical Institute) were cultured in Dulbecco's modified Eagle's medium supplemented with $10 \%$ heat-inactivated fetal bovine serum, $100 \mathrm{U} / \mathrm{ml}$ penicillin and $100 \mu \mathrm{l} / \mathrm{ml}$ streptomycin (all from Gibco-BRL,New York, NY, USA) at $37^{\circ} \mathrm{C}$ in $5 \% \mathrm{CO}_{2}$.

Design of siRNA oligos targeting the IL- 6 gene. siRNA sequences targeting the rat IL-6 gene (GenBank accession number: NM_012589; https://www.ncbi.nlm.nih. gov/genbank/) were designed using http://dharmacon. gelifesciences.com/design-center/?redirect=true. The siRNA sequences corresponding to the coding region of the IL-6 gene are shown in Table I and numbering begins from the first nucleotide of the transcription start site.

Plasmid construction. The pSUPER, pSUPER-GFP and pSUPER-siRNA-GFP vector plasmids were obtained from Dr Hua Wang (Laboratory of Cell Biology, Bureau of Life Sciences and Biotechnology, Chinese Academy of Sciences, Beijing, China). An siRNA-expressing construct was designed using a polymerase-III H1-RNA gene promoter to drive expression. Two sets of DNA oligonucleotides were used to construct the coding region of the siRNA. BglII and HindIII cleavage sites were designed in the hairpin to facilitate restriction analysis during cloning. The forward and reverse oligos included a unique 19 nucleotide target in both sense and antisense orientation, separated by a 9 nucleotide non-complementary spacer sequence (TCTCTTGA) (Fig. 1). All five pair DNA oligonucleotides generated for siRNA expression were chemically synthesized by Shanghai Sangon Biological Engineering Technology \& Services Co., Ltd. (Shanghai, China). The oligos were dissolved in sterile, nuclease-free $\mathrm{H}_{2} \mathrm{O}$ to a concentration of $3 \mathrm{mg} / \mathrm{ml}$. For the annealing reaction, $1 \mu \mathrm{l}$ of each oligo (forward and reverse) was mixed with $48 \mu \mathrm{l}$ annealing buffer. The solution was incubated at $90^{\circ} \mathrm{C}$ for $4 \mathrm{~min}$ and then at $70^{\circ} \mathrm{C}$ for $10 \mathrm{~min}$. The annealed oligos were slowly cooled to $10^{\circ} \mathrm{C}$ and the annealed sequences were inserted into the pSUPER backbone following digestion with $B g l$ II and HindIII and transformation into DH5 $\alpha$ competent cells (Tiangen Biotech Co., Ltd., Beijing, China) according to the manufacturer's instructions (OligoEngine, Seattle, WA, USA). Subsequent to amplification, all vector constructs were verified using digestion with EcoRI and XhoI followed by DNA sequencing (Sage Science, USA). The constructed plasmid vectors were referred to as pSUPER IL-6 1-5.

Transfection of C6 cells and induction. C6 cells were seeded on 24 -well plates at a density of $5 \times 10^{5}$ cells per well in a final volume of $1 \mathrm{ml}$. The cells were grown to $70 \%$ confluency. Immediately prior to transfection, the growth medium was substituted with fresh DMEM without serum and antibiotics (1 ml/well). The transfection mixtures (pSUPER IL-6 1-5 or pSUPER blank vector) were then pipetted dropwise onto the C6 cell cultures. The cells were exposed to the transfection agents for $6 \mathrm{~h}$ and the medium was then replaced with normal growth medium. The transfected cells were induced with LPS (19 ng/ml) to stimulate IL-6 secretion. Each sample was run in triplicate. C6-GFP cells transfected with the pSUPER-siRNA-GFP vector and C6 cells transfected with the pSUPER-GFP vector at the same dose were used as controls.

ELISA for IL-6 and IL-8. The supernatants of the transfected cells were collected after 24 and $48 \mathrm{~h}$ and analyzed for IL- 6 and IL-8 using ELISA according to the manufacturer's instructions (Bender Medsystems, Vienna, Austria). Statistical analysis was performed using the two-tailed t-test. The most efficiently constructed vector was selected for the animal studies in vivo.

In vivo animal experiments. Brain injury was induced using Feeney's free falling method (16). Subsequent to being weighed, rats were injected intraperitoneally with $30 \mathrm{~g} / \mathrm{l}$ sodium pentobarbital at a dose of $30 \mathrm{~g} / \mathrm{kg}$ as an anesthetic. Animals were then fixed in a stereotactic frame. A right parietal craniotomy (diameter, 
Table I. Targeted and antisense sequences of rat IL-6 siRNA.

\begin{tabular}{llll}
\hline Type & \multicolumn{1}{c}{ siRNA targeted sequence } & Antisense sequence & Start site (nt) \\
\hline T1 (pSUPER-IL-6 1) & GGACCAAGACCATCCAACT & AGTTGGATGGTCTTGGTCC & 621 \\
T2 (pSUPER-IL-6 2) & CAGCGATGATGCACTGTCA & TGACAGTGCATCATCGCTG & 298 \\
T3 (pSUPER-IL-6 3) & CTGGCAATATGAATGTTGA & TCAACATTCATATTGCCAG & 802 \\
T4 (pSUPER-IL-6 4) & GTCGGAGGCTTAATTACAT & ATGTAATTAAGCCTCCGAC & 215 \\
T5 (pSUPER-IL-6 5) & CTGGATATAACCAGGAAAT & ATTTCCTGGTTATATCCAG & 369 \\
Neg (pSUPER Neg) & GATTCAGGTGTAGAACGAG & CTCGTTCTACACCTGAATC & -
\end{tabular}

si, small interfering; IL, interleukin.

5'-GATCCCCGGACCAAGACCATCCAACTTTCAA GAGAAGTTGGATGGTCTTGGTCCTTTTTA-3' 5'-GGGCCTGGTTCTGGTATGGTTGAAAGTTCTC 'TTCAACCTACCAGAACCAGGAAAAATTCGA-3' Custom Oligos

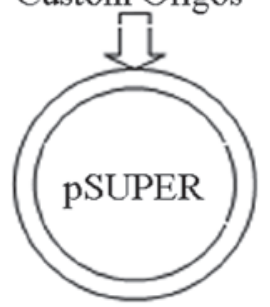

Figure 1. Schematic diagram of the construction of the pSUPER-IL-6 1 vector. The siRNA DNA fragment targeting rat IL-6 was designed and synthesized. The double strand was formed subsequent to annealing and was inserted directionally into the pSUPER plasmid following double-enzyme digestion. sh, small hairpin; IL, interleukin.

$4 \mathrm{~mm}$ ) was drilled under aseptic conditions $2 \mathrm{~mm}$ posterior to the anterior fontanelle and $2 \mathrm{~mm}$ from the skull middle line. The intact cranial dura was exposed to a freefalling weight, which produced a standardized parietal contusion by allowing a steel rod with a flat end diameter of $4 \mathrm{~mm}$, weighing $20 \mathrm{~g}$, fall onto a piston resting on the dura from a height of $30 \mathrm{~cm}$. The piston was allowed to compress the tissue to a maximum of $2 \mathrm{~mm}$. Following brain injury, 42 rats were randomly divided into three equal groups: Control, treatment and blank vector groups.

Transfection in vivo. For periodic injection of the injured area, a tubule was implanted at the injury site and secured in position using dental cement. At $-24,0,+24$ and $+48 \mathrm{~h}$ after the induction of TBI, rats were anesthetized and injected with $5 \mu \mathrm{l}$ pSUPER-IL-6 $(0.5 \mu \mathrm{g} / \mu \mathrm{l})$, pSUPER blank vector $(0.5 \mu \mathrm{g} / \mu \mathrm{l})$ or $0.9 \%$ sodium chloride over a period of $5 \mathrm{~min}$. All animals survived the intracerebral injection. At 24 and $72 \mathrm{~h}$ after the last injection, seven rats from each group were decapitated under deep anesthesia ( $80 \mathrm{mg} / \mathrm{kg}$ intraperitoneal pentobarbital). The brain was quickly removed and placed on a cooled surface. The cerebrum was coronally divided into three sections through the needle entry site and the midpoint of the posterior remnant. The first section (2-mm thick) was cut from $5 \mathrm{~mm}$ ipsilateral and contralateral of the TBI site, and then fixed using $2 \%$ paraformaldehyde and $2.5 \%$ glutaraldehyde. Frozen brain sections were prepared for electron microscopy and the two remaining sections were used for brain edema analysis and ELISA.
Analysis of brain water and sodium content. Each section was wrapped in pre-weighed aluminum foil and weighed to obtain the wet weight (WW), then dried for $72 \mathrm{~h}$ in an oven at $110^{\circ} \mathrm{C}$ and weighed again to obtain the dry weight (DW). The brain water content was calculated as the percentage change using the following formula: (WW-DW)/WW x 100.

The dehydrated brain samples were digested in $1 \mathrm{ml}$ $1 \mathrm{~N}$ nitric acid for one week. The sodium ion content was then assessed using the Inductively Coupled Plasma Emission Spectrometer ICPE-9000 (Thermo Jarrell-Ash Corporation, Franklin, MA, USA). Sodium ion levels were expressed in milliequivalents per kilogram of dehydrated brain tissue.

Statistical analysis. All data are presented as the mean \pm standard error of the mean. Data from the different animal groups were analyzed using the Student's t-test. SPSS 10.0 (SPSS Inc., Chicago, IL, USA) was used for statistical analysis. $\mathrm{P}<0.05$ was considered to indicate a statistically significant difference.

\section{Results}

Identification of recombinant plasmids. The restriction digestion enzymes EcoRI and XhoI were used to digest the empty pSUPER plasmid and the recombinant pSUPER IL-6 1-5 plasmids. Following digestion, the empty plasmid generated a 248 bp fragment, while the recombinant plasmid generated a $281 \mathrm{bp}$ fragment, which was consistent with the expected findings (Fig. 2).

Sequence determination of the inserted fragment. DNA sequencing was performed on the fragment inserted into the pSUPER-IL-6 1-5 plasmids. The findings showed that the siRNA-IL-6 sequence cloned into the pSUPER vector was the same as the designed and synthesized sequence.

IL-6 expression in C6 cells. In the positive control groups (Fig. 3A and B), following C6-GFP cell transfection with pSUPER-siRNA-GFP, the fluorescence intensity was significantly reduced (Fig. 3B), while in the negative control groups (Fig. 3C and D), following C6 cell transfection with pSUPER-GFP, the cytoplasm was observed to exhibit fluorescence (Fig. 3D).

IL-6 secretion was found to be significantly increased 24 and $48 \mathrm{~h}$ after LPS stimulation in C6 cells, peaking at 24 h. pSUPER-IL-6 1-5 were observed to affect IL-6 secretion 


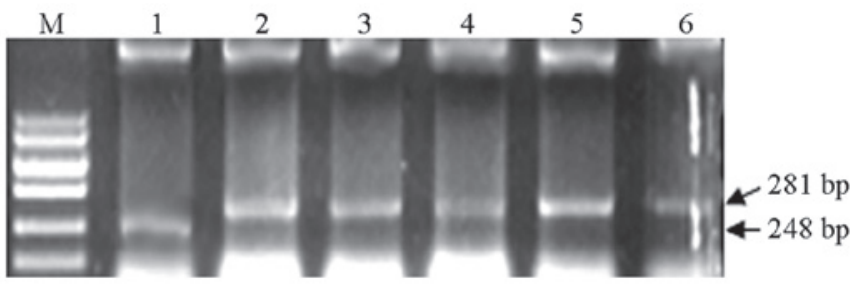

Figure 2. Identification of the recombinant plasmids following enzyme digestion. M, marker (DL2000); lane 1, pSUPER plasmid; lane 2, products of pSUPER-IL-6 1 digested using XhoI and EcoRI; lane 3, products of pSUPER-IL-6 2 digested using XhoI and EcoRI; lane 4, products of pSUPER-IL-6 3 digested using XhoI and EcoRI; lane 5, products of pSUPER-IL-6 4 digested using XhoI and EcoRI; and lane 6, products of pSUPER-IL-6 5 digested using XhoI and EcoRI. IL, interleukin.
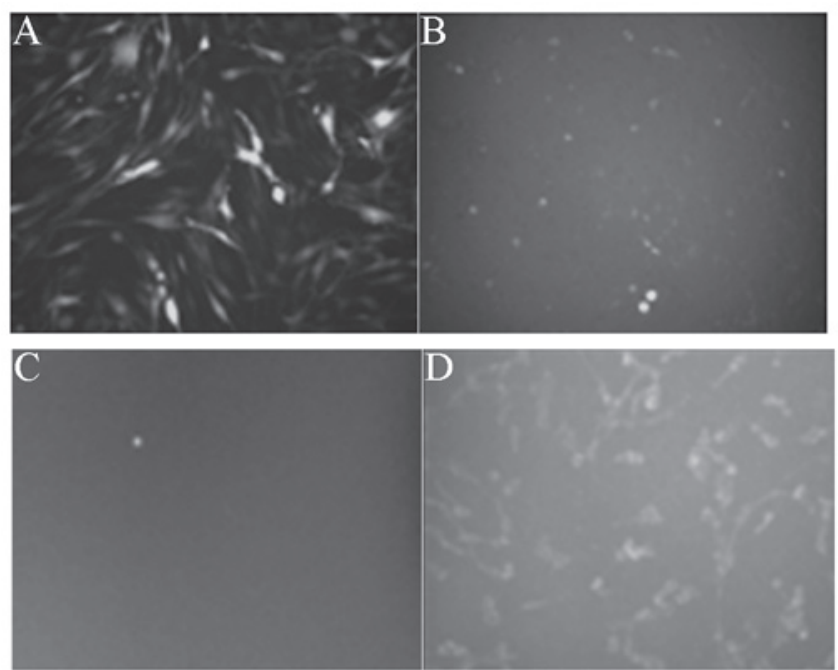

Figure 3. Inhibition of IL-6 gene expression. (A) C6-GFP cells transfected with $1 \mu \mathrm{g}$ pSUPER-siRNA-GFP plasmid as a positive control, for comparing cells prior to transfection and $24 \mathrm{~h}$ following transfection (B). C6 cells transfected with $1 \mu \mathrm{g}$ pSUPER-GFP as the negative control (C), for comparing cells prior to transfection and $24 \mathrm{~h}$ following transfection (D). (A and B) Positive controls. (C and D) Negative controls. Magnification, x100. IL, interleukin; sh, small hairpin; GFP, green fluorescent protein.

in LPS-stimulated C6 cells, with IL-6 protein levels in the culture supernatant in the pSUPER and pSUPER-IL-6 1-5 transfected cells observed to be $369 \pm 16.7,121 \pm 12,155 \pm 13.3$, $198 \pm 11.3,176 \pm 9$ and $166 \pm 11 \mathrm{pg} / \mathrm{ml}$, respectively. Moreover, the interference effect of pSUPER-IL-6 1-5 was found to be stronger $24 \mathrm{~h}$ after LPS stimulation compared with $48 \mathrm{~h}$ after LPS stimulation. ELISA revealed that pSUPER-IL-6 1 exhibited the strongest RNAi effect, inducing a $66.6 \%$ reduction in IL-6 secretion compared with the control, thus this plasmid was selected for the subsequent animal experiments (Fig. 4).

IL-8 expression in C6 cells. IL-8 basic levels were very low $(5 \pm 0.5 \mathrm{pg} / \mathrm{ml})$, however, following transfection after LPS stimulation, IL-8 levels were found to significantly increase $(377 \pm 13$ $\mathrm{pg} / \mathrm{ml})$. Compared with the control group, no significant difference was observed in IL-8 expression in LPS-stimulated C6 cells transfected with pSUPER-IL-6 1-5 (P>0.05; Fig. 5).

IL-6 expression in the brain tissue. The IL-6 levels in the peripheral brain tissues around the focal traumatic lesions after $24 \mathrm{~h}$

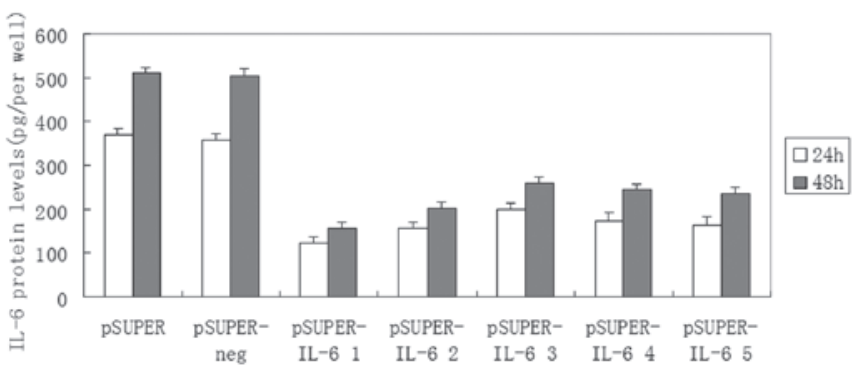

Figure 4. Inhibition of IL-6 expression using pSUPER, pSUPER-neg and pSUPER-IL-6 1-5 at 24 and $48 \mathrm{~h}$. IL, interleukin; neg, negative control.

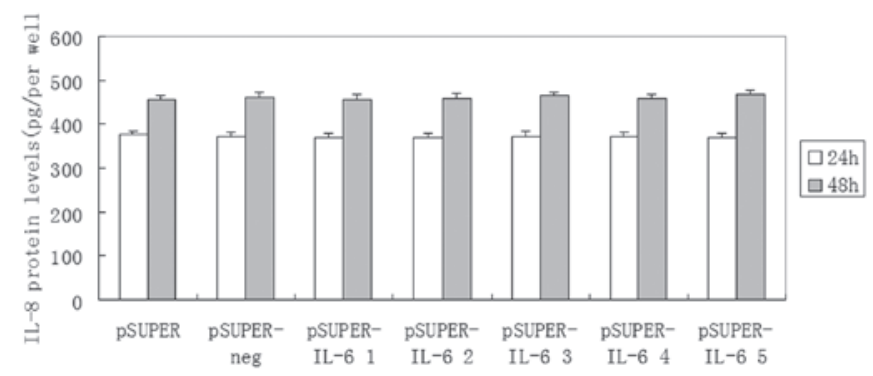

Figure 5. Effect of IL-6 RNAi on IL-8 expression using pSUPER, pSUPER-neg and pSUPER-IL-6-1-5 at 24 and 48 h. RNAi, RNA interference; IL, interleukin; neg, negative control.

were $79 \pm 4,76 \pm 5$ and $58 \pm 6.3 \mathrm{pg} / \mathrm{ml}$ in the negative control, empty plasmid and pSUPER-IL-6 1 groups, respectively. Furthermore, after $72 \mathrm{~h}$, the IL-6 levels were $72 \pm 5,71 \pm 4$ and $66 \pm 5.3 \mathrm{pg} / \mathrm{ml}$ in the negative control, empty plasmid and pSUPER-IL-6 1 groups, respectively. Compared with the control group and the empty plasmid group, IL-6 was observed to be significantly lower in the pSUPER-IL-6 1 group $(\mathrm{P}<0.05$; Fig. 6).

Brain edema. Statistically significant differences were observed in the water and sodium content in the peripheral brain tissues around the focal traumatic lesions among the control, empty plasmid and experimental groups $(\mathrm{P}<0.05$; Table II).

\section{Discussion}

The present study identified that IL-6 was overexpressed in C6 rat glioma cells treated with the microbial product LPS. This finding is in agreement with a previous study, which analyzed the association between IL-6 and post-injury treatment of C6 glioma cells (17).pSUPER-IL-6 vector-based siRNA was found to downregulate endogenous IL-6 expression in C6 rat glioma cells induced by the microbial product LPS. Furthermore, vector-based IL-6 siRNA was also found to downregulate the expression of IL- 6 in rats with TBI. Thus, the administration of siRNA may inhibit brain edema in rats with TBI.

IL-6 is a cytokine with broad immunomodulatory effects. IL-6 consists of 212 amino acids, has a molecular weight of $26 \mathrm{kDa}$ and is primarily produced by $\mathrm{T}$ and $\mathrm{B}$ lymphocytes, monocytes and endothelial cells. In the brain, IL-6 is predominantly produced by astrocytes and glial cells. Under normal circumstances, the expression of IL-6 in the brain is low, mediating a variety of physiological functions, including 
Table II. Water and sodium content of the peripheral brain tissues.

\begin{tabular}{lcc}
\hline Group & $24 \mathrm{~h}$ & $72 \mathrm{~h}$ \\
\hline Control & & \\
$\quad$ Water content (\%) & $78.68 \pm 0.76$ & $81.67 \pm 0.67$ \\
Sodium content (mmol/kg) & $671.67 \pm 40.04$ & $594.39 \pm 29.17$ \\
Empty plasmid & & \\
$\quad$ Water content (\%) & $77.49 \pm 0.59$ & $85.55 \pm 0.75$ \\
Sodium content (mmol $/ \mathrm{kg})$ & $668.13 \pm 56.11$ & $599.36 \pm 33.71$ \\
Experimental & & \\
Water content $(\%)$ & $69.03 \pm 0.55^{\mathrm{a}}$ & $75.45 \pm 0.37^{\mathrm{a}}$ \\
Sodium content $(\mathrm{mmol} / \mathrm{kg})$ & $590.24 \pm 38.54^{\mathrm{b}}$ & $510.89 \pm 39.60^{\mathrm{b}}$ \\
\hline
\end{tabular}

${ }^{\mathrm{a}} \mathrm{P}<0.05$, compared with the $\ldots$ group and ${ }^{\mathrm{b}} \mathrm{P}<0.05$ compared with the ... group.

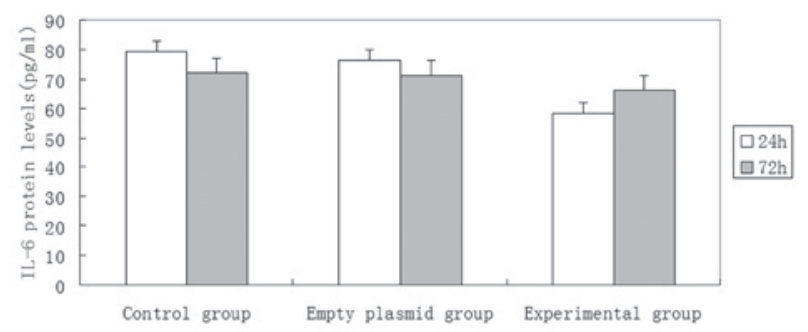

Figure 6. IL-6 expression in the brain tissue around the focal traumatic lesions after $24 \mathrm{~h}$ and $72 \mathrm{~h}$ in the control, empty plasmid and experimental groups. IL, interleukin.

central immune and nervous reparation (18). However, under pathological stress, the secretion of cerebral astrocytes and microglia increases, thus IL-6 expression increases $(2,3,19,20)$, which mediates a series of pathophysiological reactions. Such IL-6-induced pathophysiological reactions include: (i) Changes in cerebral microcirculation, resulting in increases in vascular permeability and damage to the $\mathrm{BBB}$, thus promoting the formation and progression of cerebral edema; (ii) increases in leukocyte adhesion and endothelial cells, promoting extravascular infiltration and activation of inflammatory cells $(4,19)$; (iii) increases in the proliferation and reparation of cerebral glial cells in damaged brain regions $(5,22)$; (iv) induction of complications associated with TBI, as well as multiple organ failure, for example, increases in IL-6 have a negative muscle strength effect and cytotoxic effect, which causes damage to the structure and function of the left ventricle, promoting deterioration of patient cardiac function and hemodynamics $(7,8,23,24)$; and (v) problems associated with fever and local metabolism, affecting patient prognosis and rehabilitation. As an inflammatory cytokine, IL-6 expression has been found to increase significantly in the CNS following TBI and the levels of IL-6 in the CSF has been negatively correlated with Glasgow Coma Scale and Glasgow Outcome Scale scores following injury $(9,25)$. Clinical and experimental studies have shown that during acute brain injury, IL-6 levels in the CSF increase rapidly, peaking within $24 \mathrm{~h}$ and gradually declining three days following injury, indicating that inflammatory cytokines are involved in local inflammatory responses at an early stage following the induction of TBI. Furthermore, the levels of inflammatory cytokines exhibit a significant positive correlation with disease severity and are an important indicator of condition reflection and prognosis $(11,12,26,27)$. Fundamentally, inflammation is an anti-damage response in the body; however, if the inflammatory response is too strong, severe damage to tissues and organs may be caused. Therefore, inhibiting excessive inflammatory responses has become the focus of much research $(14,28)$.

At present, studies investigating IL-6 inhibition are primarily focused on inhibiting IL-6/IL-6R or neutralizing IL-6/IL-6R antibodies and are still in the experimental stages (15-19,29-31). Based on the 3-dimensional structure of the IL- 6 protein, novel antagonist proteins have been designed, including the pertussis toxin protein (32). Furthermore, non-protein antagonists of IL-6R have been generated, including 20R,21R-epoxyresibuf ogenin-3-formate $(21,33)$. In studies on breast cancer, the IL-6 Sant 7 mutant $(22,34)$ has been used to bind IL-6R and inhibit its interactions with the glycoprotein 130 signal transduction protein, thereby inhibiting the activity of macrophages and lymphocyte-derived aromatase, reducing the synthesis of cellular estradiol. Due to the short half-life and rapid metabolism of the IL-6 antibody, as well as other factors, including immunogenicity and high cost, there are several problems associated with its clinical application. As a novel and powerful research tool, RNAi has shown great potential in the field of functional genomics, with advantages including its fast, effective, easy to operate and sequence-specific application. Therefore, the present study aimed to investigate the effect of RNAi on the regulation and inhibition of IL- 6 following transcription.

In the present study, a targeted rat IL-6 siRNA sequence was successfully designed and pSUPER-IL-6 1-5 plasmids were successfully constructed. Moreover, pSUPER-IL-6 was observed to transfect the C6 rat cerebral glioma cells effectively. LPS was used to stimulate the release of inflammatory mediators in the transfected $\mathrm{C} 6$ cells and ELISA revealed that pSUPER-IL-6 1-5 all effectively inhibited IL-6 expression, with pSUPER-IL-6 1 exerting the strongest inhibitory effect, achieving a $66.6 \%$ reduction in IL-6 secretion compared with the control. The interference effect $24 \mathrm{~h}$ after LPS induction was stronger than that $48 \mathrm{~h}$ after LPS induction, while the pSUPER empty plasmid had no effect. IL-6 and -8 expression is activated through the nuclear factor $\kappa$-light-chain-enhancer of activated B cell pathway, by IL-1 $\beta$ and TNF- $\alpha$ (35). In the present study, to investigate whether pSUPER-IL-6 transfection exerted a non-specific effect and affected IL-8 secretion, IL-8 protein levels were also assessed following transfection. Prior to LPS stimulation, IL-8 levels were observed to be very low $(5 \pm 0.5 \mathrm{pg} / \mathrm{ml})$; however, following transfection and $24 \mathrm{~h}$ after LPS stimulation, IL-8 levels were found to significantly increase $(377 \pm 13 \mathrm{pg} / \mathrm{ml})$. No significant differences in IL-8 expression were observed among the groups, indicating that the interference was specific to IL-6.

To further investigate the inhibitory effect of pSUPER-IL-6 on IL-6 protein levels in vivo, a severe rat brain injury model was generated. pSUPER-IL-6 was found to reduce IL-6 levels in the focal cerebral damage lesions. Local inflammatory response levels are directly associated with the severity of brain edema, which impacts disease progression and prognosis; therefore, the water and sodium content in the traumatic brain tissue was also 
examined. The water and sodium content of the traumatic brain tissue in the experimental group was found to be lower than that in other groups, suggesting that IL-6 was directly involved in the inflammatory response of the focal brain injury and increased brain tissue edema through the inflammatory response. Although the inflammatory response is an anti-damage response in the body, it is capable of inducing severe tissue and organ damage. Thus, controlling excessive inflammatory reactions may become a new target for studying and treating inflammation-associated diseases. In the present study, although IL-6 RNAi was observed to significantly downregulate IL-6 expression in vitro, IL-6 expression in vivo was only slightly reduced. Furthermore, only slight significant differences in brain water and sodium content were found among the groups. It is possible that the delivery of plasmids to neural cells using Lipofectamine ${ }^{\circledR} 2000$ has a poor transfection efficiency, particularly in vivo. The delivery of siRNA using viral transduction may be one way to overcome this problem. Furthermore, the continuous delivery of vectors may provide higher transfection efficiency. Brain trauma causes the expression of a variety of genes that are involved in the early post-traumatic inflammatory response (36). The large number of in vivo inflammatory cytokines form an extensive network of interactions; therefore, the identification of key inflammatory factors requires further investigation.

In conclusion, the present study showed that siRNA targeting of IL-6 mRNA using a plasmid-based system effectively sustains the knockdown of IL- 6 gene expression in C6 neural cells. However, the potential for IL-6 RNAi as a therapeutic strategy to control inflammation requires further investigation.

\section{References}

1. Dewall J: The ABCs of TBI. Evidence-based guidelines for adult traumatic brain injury care. JEMS 35: 54-61, 2010.

2. Mustafa AG and Alshboul OA: Pathophysiology of traumatic brain injury. Neurosciences (Riyadh) 18: 222-234, 2013.

3. Stein DM, Lindell A, Murdock KR, et al: Relationship of serum and cerebrospinal fluid biomarkers with intracranial hypertension and cerebral hypoperfusion after severe traumatic brain injury. J Trauma 70: 1096-1103, 2011.

4. Zhang R, Liu Y, Yan K, et al: Anti-inflammatory and immunomodulatory mechanisms of mesenchymal stem cell transplantation in experimental traumatic brain injury. J Neuroinflammation 10: 106, 2013.

5. Roberts DJ, Jenne CN, Léger C, et al: Association between the cerebral inflammatory and matrix metalloproteinase responses after severe traumatic brain injury in humans. J Neurotrauma 30 1727-1736, 2013

6. Yang SH, Gangidine M, Pritts TA, Goodman MD and Lentsch AB: Interleukin 6 mediates neuroinflammation and motor coordination deficits after mild traumatic brain injury and brief hypoxia in mice. Shock 40: 471-475, 2013.

7. Nakamura M, Okada S, Toyama Y and Okano H: Role of IL-6 in spinal cord injury in a mouse model. Clin Rev Allergy Immunol 28: 197-204, 2005.

8. Hildebrand F, Pape HC and Krettek C: The importance of cytokines in the posttraumatic inflammatory reaction. Unfallchirurg 108:793-794, 796-803, 2005 (In German).

9. Poulsen CB, Penkowa M, Borup R, et al: Brain response to traumatic brain injury in wild-type and interleukin- 6 knockout mice: a microarray analysis. J Neurochem 92: 417-432, 2005.

10. Van Wagoner NJ and Benveniste EN: Interleukin-6 expression and regulation in astrocytes. J Neuroimmunol 100: 124-139, 1999.

11. Campo S, Serlupi-Crescenzi O, Arseni B, et al: Comparative activity of Sant7 and anti-IL-6, IL-6R monoclonal antibodies in a murine model of B-cell lymphoma. Cytokine 31: 368-374, 2005.

12. Srirangan S and Choy EH: The role of interleukin 6 in the pathophysiology of rheumatoid arthritis. Ther Adv Musculoskelet Dis 2: 247-256, 2010.
13. Tuschl T, Zamore PD, Lehmann R, et al: Targeted mRNA degradation by double-stranded RNA in vitro. Genes Dev 13: 3191-3197, 1999.

14. Coelho T, Adams D, Silva A, et al: Safety and efficacy of RNAi therapy for transthyretin amyloidosis. N Engl J Med 369: 819-829, 2013.

15. Brummelkamp TR, Bernards R and Agami R: A system for stable expression of short interfering RNAs in mammalian cells. Science 296: 550-553, 2002.

16. Feeney DM, Boyeson MG, Linn RT, Murray HM and Dail WG: Responses to cortical injury: I. Methodology and local effects of contusions in the rat. Brain Res 211: 67-77, 1981.

17. Bingham D, John CM, Panter SS and Jarvis GA: Post-injury treatment with lipopolysaccharide or lipooligosaccharide protects rat neuronal and glial cell cultures. Brain Res Bull 85: 403-409, 2011.

18. Spooren A, Kolmus K, Laureys G, et al: Interleukin-6, a mental cytokine. Brain Res Rev 67: 157-183, 2011.

19. Kotzerke K, Mempel M, Aung T, et al: Immunostimulatory activity of murine keratinocyte-derived exosomes. Exp Dermatol 22: 650-655, 2013.

20. Ziebell JM and Morganti-Kossmann MC: Involvement of pro- and anti-inflammatory cytokines and chemokines in the pathophysiology of traumatic brain injury. Neurotherapeutics 7: 22-30, 2010.

21. Lu J, Goh SJ, Tng PY, Deng YY, Ling EA and Moochhala S: Systemic inflammatory response following acute traumatic brain injury. Front Biosci (Landmark Ed) 14: 3795-3813, 2009.

22. Frugier T, Morganti-Kossmann MC, O'Reilly D and McLean CA: In situ detection of inflammatory mediators in post mortem human brain tissue after traumatic injury. J Neurotrauma 27: 497-507, 2010.

23. Harhay MO, Tracy RP, Bagiella E, et al: Relationship of CRP, IL-6, and fibrinogen with right ventricular structure and function: the MESA-Right Ventricle Study. Int J Cardiol 168: 3818-3824, 2013.

24. Caselli C, D'Amico A, Caruso R, et al: Impact of normalization strategy on cardiac expression of pro-inflammatory cytokines: evaluation of reference genes in different human myocardial regions after Left Ventricular Assist Device support. Cytokine 63: 113-122, 2013.

25. Chiaretti A, Antonelli A, Mastrangelo A, et al: Interleukin-6 and nerve growth factor upregulation correlates with improved outcome in children with severe traumatic brain injury. J Neurotrauma 25: 225-234, 2008.

26. Woodcock T and Morganti-Kossmann MC: The role of markers of inflammation in traumatic brain injury. Front Neurol 4: 18, 2013.

27. Papa L, Ramia MM, Kelly JM, Burks SS, Pawlowicz A and Berger RP: Systematic review of clinical research on biomarkers for pediatric traumatic brain injury. J Neurotrauma 30: 324-338, 2013.

28. Patterson ZR and Holahan MR: Understanding the neuroinflammatory response following concussion to develop treatment strategies. Front Cell Neurosci 6: 58, 2012.

29. Erta M, Quintana A and Hidalgo J: Interleukin-6, a major cytokine in the central nervous system. Int J Biol Sci 8: 1254-1266, 2012.

30. Liu Y, Fuchs J, Li C and Lin J: IL-6, a risk factor for hepatocellular carcinoma: FLLL32 inhibits IL-6-induced STAT3 phosphorylation in human hepatocellular cancer cells. Cell Cycle 9: 3423-3427, 2010.

31. Mukaino M, Nakamura M, Yamada O, et al: Anti-IL-6-receptor antibody promotes repair of spinal cord injury by inducing microglia-dominant inflammation. Exp Neurol 224: 403-414, 2010.

32. Yang Z, Feng J, Li Y, Hu M, et al: Structure-based design and characterization of a Novel IL-6 antagonist peptide. Mol Immunol 42: 1015-1021, 2005.

33. Boos TL, Cheng K, Greiner E, Deschamps JR, Jacobson AE and Rice KC: Configurational reassignment and improved preparation of the competitive IL- 6 receptor antagonist 20R,21R-epoxyresibufogenin-3-formate. J Nat Prod 75: 661-668, 2012.

34. Purohit A, Singh A, Ghilchik MW, Serlupi-Crescenzi O and Reed MJ: Inhibition of IL-6+IL-6 soluble receptor-stimulated aromatase activity by the IL- 6 antagonist, Sant 7 , in breast tissue-derived fibroblasts. Br J Cancer 88: 630-635, 2003.

35. Ekström M, Halle M, Bjessmo S, et al: Systemic inflammation activates the nuclear factor-kappaB regulatory pathway in adipose tissue. Am J Physiol Endocrinol Metab 299: E234-E240, 2010.

36. Israelsson C, Wang Y, Kylberg A, Pick CG, Hoffer BJ and Ebendal T: Closed head injury in a mouse model results in molecular changes indicating inflammatory responses. J Neurotrauma 26: 1307-1314, 2009. 